
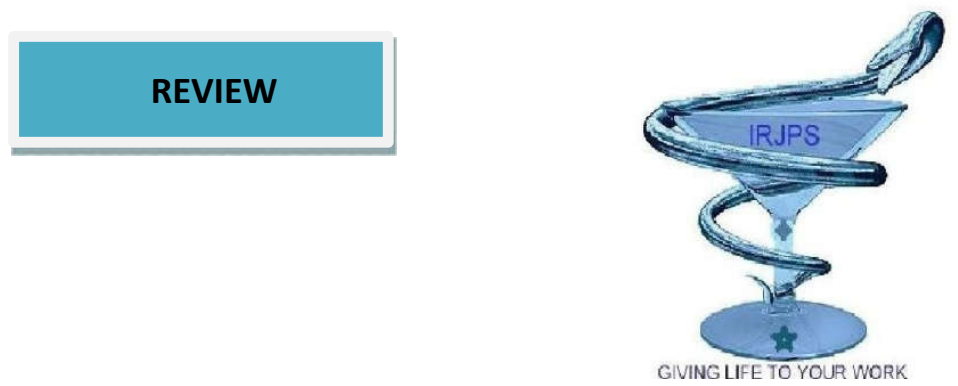

\title{
FORMULATION ASPECTS, APPROACHES AND EVALUATION OF TRANSDERMAL DRUG DELIVERY SYSTEM: A CONCISE REVIEW
}

\author{
Chandrani Roy", Arijit Gandhi \\ Department of Pharmaceutics, Bengal College of Pharmaceutical Sciences \& Research, B.R.B Sarani, Bidhannagar, \\ Burdwan, Durgapur, West Bengal 713212, India.
}

\section{Submitted on: 19.05.17; Revised on: 26.05.17; Accepted on: 30.05 .17}

\begin{abstract}
:
In the last two decades, the transdermal drug delivery system (TDDS) has become a emerging technology that offers significant clinical benefits over other dosage forms. Because transdermal drug delivery offers controlled rate of release of the drug, maintain steady state blood concentration, convenient and pain-free self-administration for patients, avoidance of hepatic first-pass metabolism and the GI tract for poorly bioavailable drugs over other routes of delivery. Characterization of transdermal patch is used to evaluate quality, size, time of onset \& duration, adhesive property, thickness, weight of patch, moisture of content, uniformity \& cutaneous toxicological studies. The present article reviews the types of transdermal patches, selection of drug candidates and polymers suitable to be formulated as transdermal system, advantages, disadvantages of formulation design and the methods of evaluation.
\end{abstract}

KEY WORDS: Transdermal drug delivery system, Bioavailability, Iontophoresis, Electroporation.

Corresponding Author: Chandrani Roy,

Telephone: (+91) 9800519269

E-mail: croy25852@gmail.com
Indian Research Journal of Pharmacy and Science; 13(2017) 963-971

Journal Home Page: https://www.irjps.in

DOI: 10.21276/irjps.2017.4.2.3 


\section{INTRODUCTION}

Despite of notable advantages of oral route of drug delivery like ease of administration, patient convenience etc., oral route possess several significant drawbacks namely poor bioavailability due to pre-systemic hepatic metabolism, the tendency to produce rapid blood level spikes (both high and low), and leading to a need for high and / or frequent dosing. To overcome these difficulties there was a need for the development of new drug delivery system, which can improve the therapeutic efficacy and safety of drugs thereby reducing dosing frequency. Transdermal delivery provides a leading alternative over injectables and oral routes by increasing patient compliance and avoiding first pass metabolism respectively. Transdermal drug delivery system (TDDS) can deliver the drugs through the skin portal to systemic circulation at a predetermined rate and maintain clinically the effective concentrations over a prolonged period of time. Transdermal therapeutic system are defined as self contained, discrete dosage form which when applied to intact skin deliver the drug through the intact skin at a control rate to the systemic circulation and maintain the drug concentration within the therapeutic window for prolonged period of time. It provides controlled, constant administration of the drug, and allows continuous input of drugs with short biological half-lives and eliminates pulsed entry into systemic circulation ${ }^{1,2}$.

\section{ADVANTAGES OF TDDS ${ }^{3,4}$}

- Avoids chemically hostile GI environment (drug degradation in acidic and basic environments is prevented).

- No GI distress and the factors like Gastric emptying, intestinal motility, transit time, do not affect this route as in oral route.

- Avoidance of significant pre-systemic metabolism (degradation in GIT or by the liver) and therefore need lower doses.

- Allows effective use of drugs with short biological half-life.

- Allow administration of drugs with narrow therapeutic window because drug levels are maintained within the therapeutic window for prolonged periods of time.
- Reduced inter and intra patient variability.

- Enhance therapeutic efficacy, reduced fluctuations (rapid blood level spikes-low and high) due to optimization of blood concentration - time profile. Reduction of dosing frequency and enhancement of patient compliance.

- Provides controlled plasma levels of very potent drugs.

- Avoids the risk and inconveniences of parenteral therapy (Painless method of drug administration).

\section{DISADVANTAGES OF TDDS ${ }^{4-6}$}

- Drugs that require high blood levels cannot be administered - limited only to potent molecules, those requiring a daily dose of 10 mg or less.

- Transdermal administration is not a means to achieve rapid bolus type drug input, rather it is usually designed to offer slow, sustained drug delivery.

- The molecular size of the drug should be reasonable that it should be absorbed percutaneously.

- Some patients develop contact dermatitis at the site of application from one or more of the system components, necessitating discontinuation.

- Tolerance inducing compounds are not an intelligent choice for this mode of administration unless an appropriate wash out period is programmed in between the dosing regimen.

\section{BASIC COMPONENTS OF TDDS ${ }^{7-9}$}

\subsection{Drug}

The drug is in direct contact with release liner. Ex: Nicotine, Methotrexate, and Oestrogen. Some of the desirable properties of a drug for transdermal delivery:

- Dose deliverable $: \leq 10 \mathrm{mg} /$ day

- Aqueous solubility $:>1 \mathrm{mg} / \mathrm{ml}$

- Lipophilicity : $\log \mathrm{P}(1-3)$

- Molecular size $:<500$ Daltons

- Melting point $:<200^{\circ} \mathrm{C}$

- Drug should not be an irritant to skin. 
- The drug should not stimulate an immune reaction in the skin.

- Along with these properties the drug should be potent, having short half life.

\subsection{Polymer matrix}

These polymers control the release of the drug from the drug reservoir. Natural polymers: shellac, gelatin, waxes, gums, starch etc., Synthetic polymers: polyvinyl alcohol, polyamide, polyethylene, polypropylene, Polyurea, polymethylmethacrylate etc.

\subsection{Permeation enhancers}

These include water, pyrolidones, fatty acids and alcohols, azone and its derivatives, alcohols and glycols, essential oils, terpenes and derivatives, sulfoxides like dimethyl sulfoximide and their derivatives, urea and surfactants. Surfactants are proposed to enhance polar pathway transport especially of hydrophilic drugs. The ability of a surfactant to alter penetration is a function of the polar head group and the hydrocarbon chain length. Anionic surfactants: sodium lauryl sulphate, Decodecylmethyl sulphoxide etc., Nonionic surfactants: Pluronic F 127, Pluronio F68, etc.

\subsection{Adhesive}

It serves to adhere the patch to the skin for systemic delivery of drug. Ex: Silicones, Polyisobutylene.

\subsection{Backing layer}

Backing layer protects patch from outer environment. Ex: Cellulose derivatives, Polypropylene silicon rubber.

\section{FACTORS EFFECTING TRANSDERMAL PERMEABILITY}

\subsection{Physico-Chemical properties of the penetrant molecules $^{11-13}$}

\subsubsection{Partition Coefficient.}

Drug having both lipid and water solubility are favorable absorbed the through the skin. A lipid/water partition coefficient equal to or more than one is generally required.

\subsection{2. $\mathrm{pH}$}

A very high and low $\mathrm{pH}$ value can be destructive to the skin. With moderate $\mathrm{pH}$ values the flux of ionisable drug can be affected by change in $\mathrm{pH}$ as these alter the ratio of charged to uncharged species and their transdermal permeability.

\subsubsection{Penetrant Concentration}

Increasing concentration of dissolved drug cause proportional increase in flux. At higher concentration, excess solid drug function as reservoir and help to maintain a constant drug concentration for a prolonged period of time.

\subsection{Physicochemical Properties of Transdermal Drug Delivery Systems ${ }^{12,13}$}

\subsubsection{Release Characteristics}

Solubility of the drug in the vehicle determines the release rate. Drug release depends on-Whether the drug molecules are dissolved or suspended in the delivery system The interfacial partition coefficient of the drug from the delivery system to skin, $\mathrm{pH}$ of the vehicle.

\subsubsection{Enhancement of Transdermal Permeation}

Majority of drugs will not penetrate the skin to reach the therapeutic level .The permeation can be improved by the addition of permeation enhancer into the system.

\subsection{Physiological and Pathological Conditions of Skin ${ }^{12,14}$}

\subsubsection{Reservoir Effect of Horny Layer}

The horny layer especially the deeper layer can sometimes act as a depot and modify the transdermal permeation of drugs. The reservoir effect is due to irreversible binding of a part of the applied drug with the skin

\subsubsection{Lipid Film}

The lipid film on the skin surface acts as a protective layer to prevent the removal of moisture from the skin and helps in maintaining the barrier function of stratum corneum.

\subsubsection{Skin Hydration}

Hydration of stratum corneum can enhance 
permeability. Skin hydration can be achieved simply by covering or occluding the skin. Increased hydration appears to open up the dense, closely packed cells of the skin and increases its porosity.

\subsubsection{Skin Temperature}

Raising the skin temperature results in an increase in the rate of skin permeation; this may be due to availability of thermal energy required for diffusivity.

\subsubsection{Regional Variation}

Difference in nature and thickness of the barrier layer of skin causes variation in permeability.

\section{TYPES OF TDDS PATCH}

\subsection{Reservoir system}

In reservoir systems the drug is enclosed between a rate controlling microporous or nonporous membrane and an impermeable backing laminate. The drug is dispersed uniformly in solid polymer matrix and suspended in a viscous liquid medium making a paste. The release rate of the drug is determined by the abrasion rate, permeability, diffusion and thickness of the membrane. The release rate is zero order process from reservoir system. The whole system is supported on the impermeable metallic backing ${ }^{15,16}$.

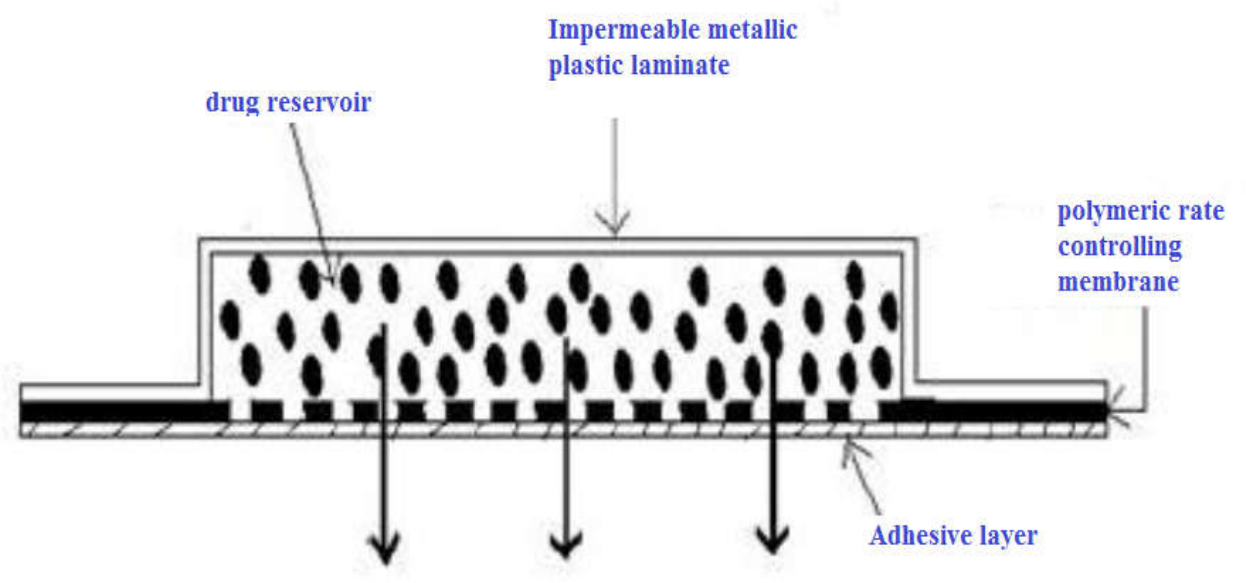

Fig 1. Reservoir type TDDS.

\subsection{Matrix diffusion system}

In matrix diffusion system drug is uniformly dispersed in hydrophilic or lipophilic polymeric material. The rate of erosion of the polymer, thickness of the layer and surface area of the film determines the release rate of the drug. No other rate controlling membrane is present in the matrix system.
Adhesive layer is spreaded around the circumference of the polymer disc instead of spreading on the surface of the patch. Matrix system of drug delivery can be modified by adding drug directly in the adhesive layer. This may be formulated in single layer drug in adhesive system or multilayer drug in adhesive system ${ }^{17,18}$.

These are also known as the monolithic systems.

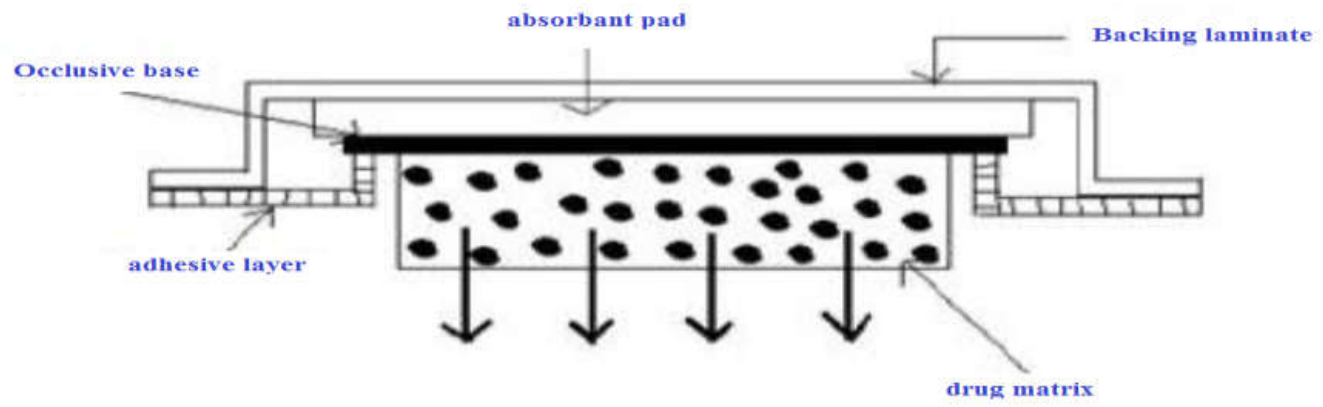

Fig 2. Matrix diffusion type TDDS. 


\subsection{Drug in adhesive system}

In this system drug is dispersed in the adhesive layer of the patch. The adhesive layer not only serves to adhere the components of the patch with the skin but also controls the rate of drug delivery to the skin. The adhesive layer is surrounded by the liner. In single layer patch a single drug in adhesive layer is present but in multilayer patch one layer is for immediate release of the drug and other layer is for controlled release of the drug ${ }^{18,19}$.

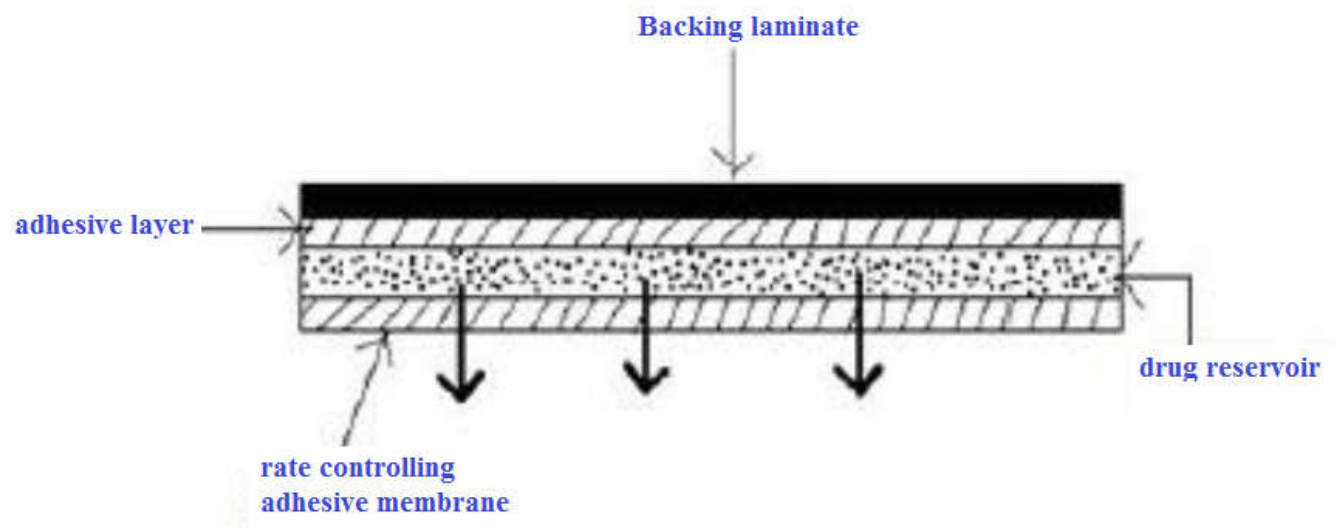

Fig 3. Drug in adhesive system.

\subsection{Microresevoir system}

The micro reservoir system is the combination of the matrix and reservoir system. In micro reservoir system the drug is first suspended in an aqueous solution of a hydrophilic polymer (e.g., PEG) and then the above suspension is mixed with a lipophilic polymer (e.g., silicon) by high shear mechanical stirrer. The cross linking of the polymer chains produced in-situ stabilizes the micro reservoir system and a medicated polymer disc of specific area and thickness is formed ${ }^{19,20}$.

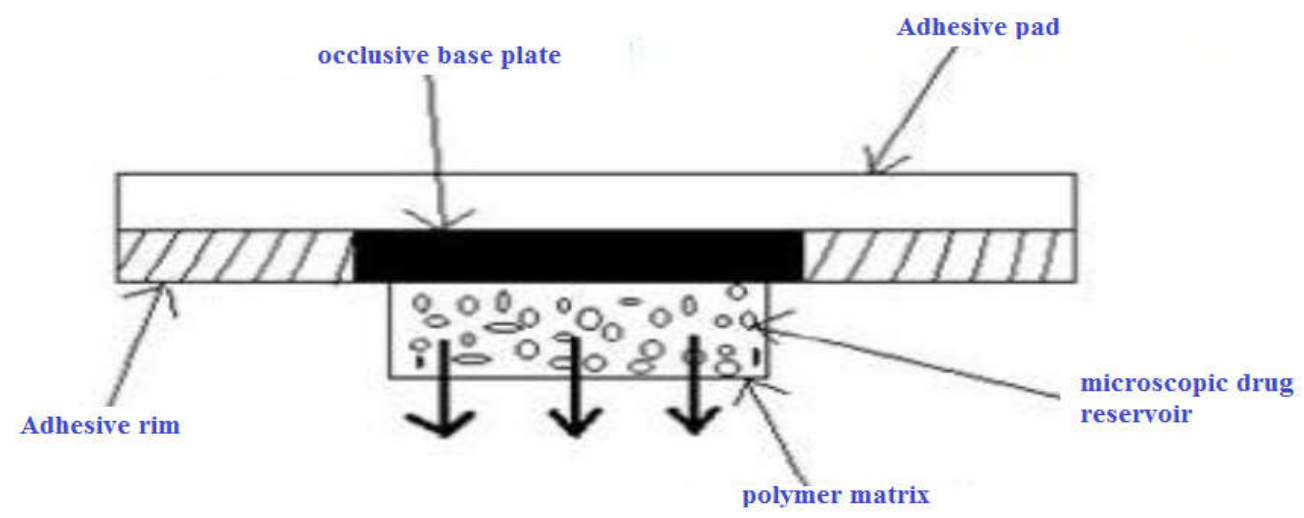

Fig 4. Microresevoir type TDDS.

\section{EVALUATION OF TDDS PATCHES ${ }^{19,21-23}$}

\subsection{Thickness}

Thickness of the transdermal patch is measured by travelling microscope, dial gauge, screw guaze or micrometer at three different points of the patch and average of the three is taken as thickness of the patch a uniformly thick patch will have an equal thickness at every point. The variation of thickness within the patch and patch to patch can be calculated.

\subsection{Folding endurance}

A strip of specific area is cut evenly and repeatedly folded at the same place till it broke. The number of 
times the film is folded at the same place without breaking gives the value of the folding endurance.

\subsection{Tensile strength}

The tensile strength of the patch was evaluated by using the tensiometer. It consists of two load cell grips. The lower one was fixed and upper one was movable. Film strips with specific dimensions of were fixed between these cell grips, and force was gradually applied till the film broke. The tensile strength was taken directly from the dial reading in $\mathrm{kg}$.

\subsection{Swellability}

The patches of specific area was weighed and put in a petridish containing $10 \mathrm{ml}$ of double distilled water and were allowed to imbibe. Increase in weight of the patch was determined at preset time intervals, until a constant weight was observed. The degree of swelling (S) was calculated using the formula: S (\%) $=(\mathrm{Wt}-\mathrm{Wo}) / \mathrm{Wo} \times 100$ Where $\mathrm{S}$ is percent swelling, $\mathrm{Wt}$ is the weight of patch at time $\mathrm{t}$ and Wo is the weight of patch at time zero.

\subsection{Drug content determination}

An accurately weighed portion of film is dissolved in $100 \mathrm{ml}$ of suitable solvent in which drug is soluble and then the solution is shaken continuously for 24 hrs in shaker incubator. Then the whole solution is sonicated. After incubation and subsequent filtration, drug in solution is estimated spectrophotometrically by appropriate dilution.

\subsection{Percentage Moisture content}

The prepared films are weighed individually and kept in a desiccators containing calcium chloride at room temperature for $24 \mathrm{hrs}$. The films are weighed again after a specific interval until they show a constant weight. The percent moisture content is calculated using formula. Percentage moisture content $=[($ Initial weight- Final weight)/ Final weight] X 100.

\subsection{In vitro drug release studies}

The paddle over disc method can be employed for assessement of the release of the drug from the prepared patches. Dry films of known thickness is to be cut into definite shape, weighed, and fixed over a glass plate with an adhesive. The glass plate was then placed in a $500 \mathrm{ml}$ of the dissolution medium or phosphate buffer (pH 7.4). The paddle was then set at a distance of $2.5 \mathrm{~cm}$ from the glass plate and operated at a speed of $50 \mathrm{rpm}$. Samples can be withdrawn at appropriate time intervals upto $24 \mathrm{hrs}$ and analysed by UV spectrophotometer or HPLC. The experiment is to be performed in triplicate and the mean value can be calculated.

\subsection{In vitro skin permeation studies}

An in vitro permeation study can be carried out by using diffusion cell. Full thickness abdominal skin of male Wistar rats weighing 200-250 gm. Hair from the abdominal region is to be removed carefully by using a electric clipper, the dermal side of the skin was thoroughly cleaned with distilled water to remove any adhering tissues or blood vessels, equilibrated for an hour in dissolution medium or phosphate buffer pH 7.4 before starting the experiment. The isolated rat skin piece is to be mounted between the compartments of the diffusion cell, with the epidermis facing upward into the donor compartment. Sample volume of definite volume is to be removed from the receptor compartment at regular intervals, and an equal volume of fresh medium is to be replaced. Samples are to be filtered through filtering medium and can be analysed spectrophotometrically or HPLC. Flux can be determined directly as the slope of the curve between the steady state values of the amount of drug permeated vs. time in hrs and permeability coefficients were deduced by dividing the flux by the initial drug load.

\subsection{Skin irritation study}

Skin permeation and sensitization testing can be performed on healthy rabbits. The dorsal surface of the rabbit is to be cleaned and remove the hair from the clean dorsal surface by using rectified spirit and the representative formulations can be applied over the skin. The patch is to be removed after $24 \mathrm{hrs}$ and the skin is to be observed and classified into 5 grades on the basis of the severity of skin injury.

\section{APPROACHES OF TDDS}

The application of the transdermal patch and the flow of the active drug constituent from the patch to the circulatory system via skin occur through various methods: 


\subsection{Iontophoresis}

Iontophoresis is a method of transferring substances across the skin by applying an electrical potential difference. It promotes the transfer of charged ionic drugs and possibly high molecular weight substances such as peptides. Electric current is applied through two electrodes, placed in the patient's skin. The first, or donor, electrode (cathode) delivers the negatively charged therapeutic agent (e.g., an organic acid), whereas the second or receptor, electrode (anode) serves to close the circuit. This setup is named cathodal iontophoresis. For positively charged drugs (e.g., amines or peptides), the cell arrangement is reversed called anodal iontophoresis. The silver (anode) and silver chloride (cathode) electrode system utilized in both types of iontophoresis. Three main mechanisms enhance molecular transport: i) Charged species are driven primarily by electrical repulsion from the driving electrode, ii) Flow of electric current may increase the permeability of skin and iii) Electroosmosis may affect uncharged molecules and large polar peptides ${ }^{19}$.

\subsection{Phonophoresis}

In this process ultrasonic energy is used. The medicine is placed on the skin over the area to be treated and massaging the site with an ultrasound source. The ultrasonic energy disturbs the lipid packing in the intercellular spaces of the stratum corneum by heating and cavitations effects, and thus enhances drug penetration into the tissue ${ }^{24}$.

\subsection{Electroporation}

This method involves application of short, highvoltage electrical pulses to the skin. After electroporation, the permeability of the skin for diffusion of drugs is increased by 4 orders of magnitude. The electrical pulses are believed to form transient aqueous pores in the stratum corneum, through which drug transport occurs. It is safe and the electrical pulses can be administered painlessly using closely spaced electrodes to constrain the electric field within the nerve-free stratum corneum ${ }^{25}$.

\subsection{Use of microscopic projection}

Transdermal patches with microscopic projections called microneedles were used to facilitate transdermal drug transport. Needles ranging from approximately $10-100 \mu \mathrm{m}$ in length are arranged in arrays. When pressed into the skin, the arrays make microscopic punctures that are large enough to deliver macromolecules, but small enough that the patient does not feel the penetration or pain. The drug is surface coated on the microneedles to aid in rapid absorption. They are used in development of cutaneous vaccines for tetanus and influenza ${ }^{24}$.

\subsection{Skin Abrasion}

The abrasion technique involves the direct removal or disruption of the upper layers of the skin. These devices are based on techniques employed by dermatologists for superficial skin resurfacing which are used in the treatment of acne, scars, hyperpigmentation and other skin blemishes ${ }^{19,25}$.

\section{CONCLUSIONS}

In recent years it has been shown that the skin is a useful route for drug delivery to the systemic circulation. Transdermal drug delivery system includes all topically administered drug formulations intended to deliver the active ingredients into the circulation. Transdermal delivery of drugs is likely to continue to increase. Research is being performed to increase safety and efficacy. Now days transdermal drug delivery system is widely recognized as one of the most reliable, appealing as well as effective technique of drug delivery. Future development of transdermal therapeutic system involves improvement of presently available devices to provide optimum blood levels, availability of better skin permeability enhancers and novel formulation approaches.

\section{REFERENCES}

1. Singh MC, Naik AS, Sawant SD., Transdermal Drug Delivery Systems with major emphasis on Transdermal patches : A Review., Journal of Pharmacy Research, 2010; 3(10): 2537-2543.
2. Sakalle P, Dwivedi S, Dwivedi A., Design, Evaluation, Parameters and Marketed Products of transdermal patches: A Review., Journal of Pharmacy Research, 2010; 3(2): 235-240. 
3. Sharma N, Agrawal G, Rana A, Alibhat Z, Kumar D., A Review: Transdermal Drug Delivery System: A Tool For Novel Drug Delivery System., International Journal of Drug Development \& Research, 2011; 3(3): 70-84.

4. Kurz A, Farlow M, Lefevre G., Pharmacokinetics of a novel transdermal rivastigmine patch for the treatment of Alzheimer's disease: a review., International Journal of Clinical Practice, 2009; 63(5): 799-805.

5. Panner Selvam R, Kumar Singh A, Sivakumar T., Transdermal drug delivery systems for antihypertensive drugs - A review., International Journal of Pharmaceutical And Biomedical Research, 2010; 1(1): 1-8.

6. Gaur PK, Mishra S, Purohit S, Dave K.., Transdermal Drug Delivery System: A Review., Asian Journal of Pharmaceutical and Clinical Research, 2009; 2(1): 14-20.

7. Jalwal P, Jangral A, Dahiya L, Sangwan Y, Saroha R., A Review on Transdermal Patches., The Pharm. Res., 2010; 3(2): 139-149.

8. Parivesh S, Sumeet D, Abhishek D., Design, Evaluation, Parameters and Marketed Products of transdermal patches., A Review. J. Pharm. Res., 2010; 3(2): 235- 240.

9. Alexander A, Dwivedi S, Tripathi DK.., Approaches for breaking the barriers of drug permeation through transdermal drug delivery., Journal of Controlled Release, 2012;164:26-40.

10. Prausnitz MR, Langer R., Transdermal drug delivery., Nat Biotechnol., 2008; 26(11):1261-1268.

11. Keleb E, Sharma RK, B mosa E, Z aljahwi A., Transdermal Drug Delivery System Design and Evaluation., Int. J. of Advances in Pharm. Sci., 2012;1;201-211.

12. Vishwakarma SK, Niranjan SK, Irchhaiya R, Kumar N, Akhtar A., A Novel transdermal drug delivery system., International Journal of research of pharmacy, 2012; 3(8):39-44.
13. Shingade GM, Aamer Q, Sabale PM, Gramprohit ND, Gadhave MV, Jadhv SL, Gaikwad DD., Review on: recent trend on transdermal drug delivery system., Journal of Drug Delivery \& Therapeutics, 2012; 2 (1): 66-75.

14. Hanumanaik M, Patil U, Kumar G, Patel SK, Singh I, Jadatkar K., Design, evaluation and recent trends in transdermal drug delivery system: a review., International Journal of pharmaceutical sciences and research, 2012; 3(8):2393-2406.

15. Rastogi V, Yadav P. Transdermal drug delivery system: An overview., Asian Journal of Pharmaceutics, 2012; 6(3):161-170.

16. Arunachalam A, Karthikeyan M, Kumar VD, Prathap M, Sethuraman S, Ashutoshkumar S, Manidipa S., Transdermal Drug Delivery System: A Review., Current Pharma Research, 2010; 1(1) : 7081.

17. Kapoor D, Patel M, Singhal M., Innovations in Transdermal drug delivery system., International Pharmaceutica Sciencia, 2011; 1(1): 54-61.

18. Keleb E, Sharma RK, MosaEsmaeil B, Z aljahwi A., Review on Transdermal Drug Delivery SystemDesign and Evaluation., International Journal of Advances in Pharmaceutical Sciences, 2011; 1:201211.

19. Patel D, Sunita A, Parmar B, Bhura N., Transdermal Drug Delivery System: A Review., The Pharma Innovation, 2012; 1(4): 66-75.

20. Sharma ,N. , Agarwal, G., Rana, A.C., Ali Bha,tZ.,Kumar ,D.,2011. A Review: Transdermal Drug Delivery System: A Tool For Novel Drug Delivery System, International Journal of Drug Development \& Research 3(3), 70-84.

21. Gandhi A, Jana S, Paul A, Sheet S, Nag R, Sen KK., Metoprolol tartrate Containing Glutaraldehyde Cross-linked Chitosan- Polyvinyl pyrrolidone Matrix Transdermal Patch: Preparation and Characterization., Journal of PharmaSciTech, 2014; 3(2):72-76. 
22. Maji P, Gandhi A, Jana S, Maji N., Preparation and Characterization of Maleic Anhydride CrossLinked Chitosan-Polyvinyl Alcohol Hydrogel Matrix Transdermal Patch., Journal of PharmaSciTech, 2013; 2(2):62-67.

23. Kumar JA, Pullakandam N, Prabu SL, Gopal V., Transdermal drug delivery system: an overview., International Journal of Pharmaceutical Sciences Review and Research, 2010; 3(2): 49-54.
24. Bathe R, Kapoor R., Transdermal drug delivery system: formulation, development and evaluation-An overview., International Journal of Biomedical and Advance Research, 2015; 6(01): 1-10.

25. Sachan R, Bajpai M., Transdermal drug delivery system: A Review., Int. J. Res. Dev. Pharm. L. Sci., 2013; 3(1): 748-765. 